\title{
Cross-cultural adaptation and validation of the VISA-A questionnaire for German-speaking Achilles tendinopathy patients Heinz Lohrer*† and Tanja Nauck ${ }^{\dagger}$
}

\author{
Address: Institute for Sports Medicine, Otto-Fleck-Schneise 10, D-60528 Frankfurt am Main, Germany \\ Email: Heinz Lohrer* - lohrer@smi-frankfurt.de; Tanja Nauck - nauck@smi-frankfurt.de \\ * Corresponding author †Equal contributors
}

Published: 30 October 2009

BMC Musculoskeletal Disorders 2009, 10:134 doi:10.1186/147|-2474-10-134

This article is available from: http://www.biomedcentral.com/I47/-2474//0/I34

(C) 2009 Lohrer and Nauck; licensee BioMed Central Ltd.

This is an Open Access article distributed under the terms of the Creative Commons Attribution License (http://creativecommons.org/licenses/by/2.0), which permits unrestricted use, distribution, and reproduction in any medium, provided the original work is properly cited.
Received: 19 May 2009
Accepted: 30 October 2009

\begin{abstract}
Background: Achilles tendinopathy is the predominant overuse injury in runners. To further investigate this overload injury in transverse and longitudinal studies a valid, responsive and reliable outcome measure is demanded. Most questionnaires have been developed for English-speaking populations. This is also true for the VISA-A score, so far representing the only valid, reliable, and disease specific questionnaire for Achilles tendinopathy. To internationally compare research results, to perform multinational studies or to exclude bias originating from subpopulations speaking different languages within one country an equivalent instrument is demanded in different languages. The aim of this study was therefore to cross-cultural adapt and validate the VISA-A questionnaire for German-speaking Achilles tendinopathy patients.
\end{abstract}

Methods: According to the "guidelines for the process of cross-cultural adaptation of self-report measures" the VISA-A score was cross-culturally adapted into German (VISA-A-G) using six steps: Translation, synthesis, back translation, expert committee review, pretesting $(n=77)$, and appraisal of the adaptation process by an advisory committee determining the adequacy of the cross-cultural adaptation. The resulting VISA-A-G was then subjected to an analysis of reliability, validity, and internal consistency in 30 Achilles tendinopathy patients and 79 asymptomatic people. Concurrent validity was tested against a generic tendon grading system (Percy and Conochie) and against a classification system for the effect of pain on athletic performance (Curwin and Stanish).

Results: The "advisory committee" determined the VISA-A-G questionnaire as been translated "acceptable". The VISA-A-G questionnaire showed moderate to excellent test-retest reliability $(I C C=0.60$ to 0.97$)$. Concurrent validity showed good coherence when correlated with the grading system of Curwin and Stanish (rho $=-0.95$ ) and for the Percy and Conochie grade of severity (rho 0.95). Internal consistency (Cronbach's alpha) for the total VISA-A-G scores of the patients was calculated to be 0.737 .

Conclusion: The VISA-A questionnaire was successfully cross-cultural adapted and validated for use in German speaking populations. The psychometric properties of the VISA-A-G questionnaire are similar to those of the original English version. It therefore can be recommended as a sufficiently robust tool for future measuring clinical severity of Achilles tendinopathy in German speaking patients. 


\section{Background}

Achilles tendinopathy is the most common differential diagnosis of posterior heel pain [1-4]. Achilles tendinopathy is the predominant overuse injury in runners with a prevalence of $10 \%$ [5] and an incidence of $0.01 / 1000 \mathrm{~km}$ [6].

The worth of conservative and operative treatment options is still under debate [7]. To further investigate these issues in transverse and longitudinal studies a valid, responsive, and reliable outcome measure is demanded. For this reason an international group of experts from the Victorian Institute of Sports Assessment developed the VISA-A (the second A stands for "Achilles tendon") questionnaire [8].

"The VISA-A is an easily self-administered questionnaire that evaluates symptoms and their effect on physical activity. It can be used to compare different populations with Achilles tendinopathy, and facilitate comparisons between studies. It can be used to determine the patient's clinical severity and provide a guideline for treatment as well as for monitoring the effect of treatment [9]." Pain (questions 1-3), function (questions 4-6), and activity (questions 7 and 8) are the domains under consideration [8]. Maximum score of each item one to seven is 10, while question 8 yields a maximum of 30 . Consequently a summed maximum score of 100 represents an asymptomatic person [8].

The VISA-A questionnaire represents the only valid, reliable, and disease specific score to measure the condition of the Achilles tendon. Meanwhile it achieved wide acceptance [9-16]. However, as most questionnaires it has been developed for an English-speaking population. To internationally compare research results, to perform multinational studies or to minimize bias originating from subpopulations speaking different languages within one country a unique protocol of forward and back translations and cultural adaptations as well as "verification of the scaling requirements (item performance, item weights) and validation of and establishing normative values for the new version" are required [17]. This procedure has already been performed and is published for the Swedish [9] and Italian [12] version of the VISA-A questionnaire. Cross-cultural adaptations to Spanish, Portuguese, and Flemish language have been done (Karim Khan personal communication, 2009) but were not reported in journals available in Medline/PubMed. The aim of this study was therefore to cross-culturally adapt and validate the VISA-A questionnaire for German-speaking Achilles tendinopathy patients.

\section{Methods}

The study was approved by the local ethics committee.
According to the "guidelines for the process of cross-cultural adaptation of self-report measures" the VISA-A score was cross-culturally adapted into German (VISA-A-G) in six steps [17].

Step I: Initial translation from English to German (forward translation) was performed by three independent translators with German mother tongue. An orthopaedic surgeon specialised in foot, ankle, and Achilles tendon, and a sport scientist both were aware of the concepts being examined in the questionnaire. A third translator without medical background ("naïve translator") was not familiar with the questionnaire's concepts. Written reports were made to indicate rationales for decision making, linguistic difficulties and problems with regards to content.

Step II: Considering the original VISA-A the resulting three German versions were synthesized during a consensus meeting of the three translators. A written report documented the procedure.

Step III: Back translation of the preliminarily VISA-A-G questionnaire was then conducted by two American English native speakers fluid in German but without medical background and blind to the original VISA-A.

Step IV: An expert committee was constituted including forward and back translators, a health professional, and a language professional. This panel developed the prefinal VISA-A-G version by consensus discussion with respect to semantic, idiomatic, experiential, and conceptual equivalence based on all previous material (original, target and back translated questionnaires including the respective reports).

Step V (pretesting): The prefinal questionnaire was filled out by a cohort of 35 male and 42 female persons (asymptomatic persons). These persons were then asked regarding their individual understanding of the questionnaire's items. The interviewer prepared a report on the test subjects, understanding of the items, and their decision making. Again the questionnaire was adapted respectively resulting in a final version.

Step VI: The final version of the VISA-A-G questionnaire and all reports and forms were then subjected to a review done by the developers of the VISA-A-G questionnaire for appraisal of the adaptation process.

The final VISA-A-G questionnaire was then subjected to psychometric testing including an analysis of reliability and validity in 30 Achilles tendinopathy patients and 79 asymptomatic people. Internal consistency was evaluated in 30 Achilles tendinopathy patients. 
This was done following closely the procedure described in the original VISA-A report [8].

Test subjects: For reliability and validity testing four groups of test subjects were defined according to the status of their Achilles tendon. Group I included 15 preoperative Achilles tendinopathy patients selected prospectively from the own center. Group II consisted of 15 Achilles tendinopathy patients conservatively treated. These patients were recruited from review of the most recent charts in our sports medicine center. 48 Frankfurt/Main university pharmacology students (one class preparing for their examinations) without Achilles tendon complaints constituted group III. Group IV was formed by 31 members of three local running clubs serving as non injured but active controls. That population was recruited by direct contact during their practise.

Inclusion criteria for all four groups were: age over 18 years, willingness to take part in this project, and ability to give written informed consent. Contrasting to the original VISA-A publication [8] only patients suffering from unilateral Achilles tendinopathy were included (group I and II). Achilles tendinopathy was defined as tenderness of the midportion of the Achilles tendon 2 to $7 \mathrm{~cm}$ proximal to the Achilles tendon insertion and a history of load induced pain in this area [2-4]. This clinical diagnosis includes degenerative Achilles tendon lesions, Achilles tendon partial tears on a microscopic level, and paratendinosis or combinations of these. For the control groups (group III and VI) subjects with previous Achilles tendon injuries were included, if they did not complain any actual symptoms or functional deficiencies. Persons with inadequate compliance and pregnant or nursing women were excluded from participating in any of the four groups. Patients (group I and II) with bilateral symptoms, subtotal or complete Achilles tendon tears, insertional Achilles tendinopathy, Haglund's disease, and relevant injuries or previous surgery of the lower extremity, radicular and pseudoradicular induced pain were excluded.

To document the individual level of activity the ankle activity score which is a reliable, valid, and sensitive measure [18] was calculated for all test subjects.

For concurrent validity testing all participants $(\mathrm{n}=109)$ completed the VISA-A-G questionnaire and Achilles tendinopathy severity was additionally rated by an orthopaedic surgeon specialized in Achilles tendon disorders (HL) using a generic tendon grading system [19] and a "classification system for the effect of pain on athletic performance" [20]. Even if the clinimetric properties of these tools are not formally validated so far they have been used as a standard for concurrent validity testing in previous VISAA validation research $[8,9,12]$. Percy and Conochie cate- gorized pain and function following surgery of Achilles tendon tears: "Excellent" means full function and no residual disability. "Good" is defined as full function with no real limitation of activities and minor pain. A "fair" result is represented by some limitation of activities. Severe weakness and marked limping indicates a "poor" result [19]. Curwin and Stanish assessed six degrees of reported exercise induced tendon pain and the level of sports performance: $1=$ no pain and unrestricted level of sports performance; 2 = pain only with extreme exertion and unrestricted level of sports performance; $3=$ pain with extreme exertion and 1 to 2 hours afterward and normal or slightly decreased level of sports performance; $4=$ pain during and after any vigorous activities and somewhat decreased level of sports performance; 5 = pain during activity forcing termination and markedly decreased level of sports performance; 6 = pain during daily activities and unable to perform sport [20].

Besides this, VISA-A-G results for patients expected to present moderate symptoms (group II) and patients probably suffering the most severe symptoms (group I) were compared with the controls (group III and IV).

Next, the VISA-A-G ratings from this study were compared with the respective VISA-A group values presented in the original publication [8].

For reliability testing a subset of subjects were included. For test retest reliability all conservative patients $(n=15)$, all students $(\mathrm{n}=48)$, and all joggers $(\mathrm{n}=31)$ were evaluated by the VISA-A-G questionnaire two times within one week. Intertester reliability was assessed by comparing the results when both authors administered the VISA-A-G questionnaire independently in a one to seven days interval to five participants from the students and conservative Achilles tendinopathy group respectively.

\section{Statistical analysis}

Statistics were performed using descriptive data analysis as mean, standard deviation and 95\% confidence interval. The Kolmogorov-Smirnov test was applied to check out for normal distribution. Concurrent validity was calculated by Spearman's rank correlation coefficient between the VISA-A-G scores and the two standard scoring scales $[19,20]$. Comparison of the VISA-A-G group scores with the originial VISA-A [8] was performed using a two sample t-test since only means and standard deviations were available from the original report. Reliability testing was done by Spearman's rho, Inter-Class Correlation Coefficient (ICC) and Wilcoxon paired test for nonparametric data. Internal consistency for the total score was calculated using Cronbach's alpha. Significance level was set at $\mathrm{p}<0.05$. 


\section{Results \\ Cross-cultural adaptation of the VISA-A to the VISA-A-G questionnaire}

With regards to content one of the forward translators had troubles to understand the meaning of the predetermined different answers and scorings to question 8 ("If you have no pain while undertaking Achilles tendon loading sports, for how long can you train/practise?"): If someone would have no sport related Achilles tendon pain, (s)he will be able to train as long as (s)he likes. Considering this, all persons free of Achilles tendon pain would consequently be expected to score 30 points. In this sense this question seemed to be irrelevant for patients suffering from Achilles tendinopathy. We discussed this problem with the developers of the original VISA-A questionnaire by E-mail who cleared this issue: if someone is just recovering from Achilles tendinopathy, (s)he may be on reduced training at first, say 15 minutes necessitating further answer categories (Karim Khan personal communication, 2009). Besides this there were no relevant problems reported from the three forward and also from the back translators and minor linguistic discrepancies could easily be resolved in the consensus and expert committee meeting respectively. Prefinal VISA-A-G questionnaire testing revealed some difficulties in comprehension of the questions 1 and 6. According to the Swedish translation [16] the boxes were marked with the related minutes/times to complete the final VISA-A-G version. Finally the developers approved the VISA-A-G questionnaire [see Additional file 1] and the cross-cultural adaptation process.

\section{VISA-A-G test population}

The tested groups were not homogeneous comparing age (Table 1). Asymptomatic students (Group III) were signif- icantly younger than the subjects in the three other groups (each $\mathrm{p}<0.001$ ) and preoperative patients were older than the joggers group ( $\mathrm{p}=0.032)$. The ankle activity mean score did not differ significantly $(\mathrm{p}=0.733$ to 0.999) between the four test groups (Table 1). Achilles tendinopathy severity as measured with the generic tendon grading system [19] and the "classification system for the effect of pain on athletic performance" [20] did not differ comparing the preoperative with the conservative patients, the students, and the joggers group respectively (each $\mathrm{p}=0.000$ ). There was no difference found between the jogger's and the student's groups ( $\mathrm{p}=0.864$ and 0.998; Table 1).

\section{VISA-A-G score results}

Related to controls (group III and IV) VISA-A-G scores for patients (group I and II) were significantly lower ( $\mathrm{p}=$ 0.000). VISA-A-G questionnaire outcome was not different comparing the asymptomatic control groups III with IV $(p=0.347)$ while the symptomatic groups I with II differed significantly $(\mathrm{p}=0.000)$. The preoperative and the conservative group each were significantly different from both control groups (each $\mathrm{p}=0.000$ Table 1 , Figure 1 ).

\section{Validity testing}

Comparing the VISA-A-G ratings from this study with the respective VISA-A group values presented in the original publication [8] revealed no significant differences comparing the healthy and the preoperative patient groups. Comparison between the "conservative" groups just reached the significance level (Table 2 ).

The VISA-A-G questionnaire correlated significantly with both tendon grading systems (concurrent validity). For

Table I: Age, outcome of the VISA-A-G, Ankle Activity Score [18] Achilles tendinopathy severity as measured with a generic tendon grading system [19] and the "classification system for the effect of pain on athletic performance"[20] for the study population.

\begin{tabular}{|c|c|c|c|c|c|c|c|c|c|c|c|c|c|c|c|}
\hline & \multicolumn{3}{|c|}{ Age [years] } & \multicolumn{3}{|c|}{ VISA-A-G Score } & \multicolumn{3}{|c|}{$\begin{array}{c}\text { Ankle Activity Score } \\
{[18]}\end{array}$} & \multicolumn{3}{|c|}{$\begin{array}{l}\text { Percy \& Conochie } \\
\text { Score [19] }\end{array}$} & \multicolumn{3}{|c|}{$\begin{array}{l}\text { Curwin \& Stanish } \\
\text { Score [20] }\end{array}$} \\
\hline & Mean & SD & $\begin{array}{c}95 \% \\
\mathrm{Cl}\end{array}$ & Mean & SD & $\begin{array}{c}95 \% \\
\mathrm{Cl}\end{array}$ & Mean & SD & $\begin{array}{l}95 \% \\
\mathrm{CI}\end{array}$ & Mean & SD & $95 \% \mathrm{Cl}$ & Mean & SD & $\begin{array}{l}95 \% \\
\mathrm{Cl}\end{array}$ \\
\hline $\begin{array}{l}\text { Preoper } \\
\text { ative } \\
\text { Patients }\end{array}$ & 47,8 & 11,4 & $\begin{array}{c}41,5- \\
54,1\end{array}$ & 44,9 & 14,2 & $\begin{array}{l}37,0- \\
52,7\end{array}$ & 4,6 & 1,5 & $3,8-5,4$ & 1,5 & 0,5 & $1,2-1,8$ & 5,3 & 0,7 & $4,9-5,7$ \\
\hline $\begin{array}{l}\text { Conser } \\
\text { vative } \\
\text { Patients }\end{array}$ & 44,6 & 14,0 & $\begin{array}{c}36,9- \\
52,4\end{array}$ & 73,1 & 13,5 & $\begin{array}{l}65,6- \\
80,5\end{array}$ & 5,2 & 1,6 & $4,3-6,1$ & 2,3 & 0,8 & I,8-2,7 & 3,3 & 1,2 & $2,7-4,0$ \\
\hline $\begin{array}{l}\text { Student } \\
s\end{array}$ & 21,0 & 3,9 & $\begin{array}{l}20,0- \\
22,1\end{array}$ & 98,0 & 7,1 & $\begin{array}{l}95,9- \\
100,0\end{array}$ & 4,9 & 2,1 & $4,3-5,5$ & 3,8 & 0,4 & $3,7-4,0$ & 1,2 & 0,7 & $1,0-1,4$ \\
\hline Joggers & 39,3 & 11,7 & $\begin{array}{r}35,0- \\
43,6\end{array}$ & 99,2 & 2,0 & $\begin{array}{l}98,5- \\
99,9\end{array}$ & 4,7 & 0,5 & $4,5-4,9$ & 3,7 & 0,5 & $3,6-3,9$ & 1,3 & 0,4 & $I, I-I, 4$ \\
\hline
\end{tabular}

$\mathrm{SD}=$ standard deviation $\mathrm{Cl}=95 \%$ confidence interval. 


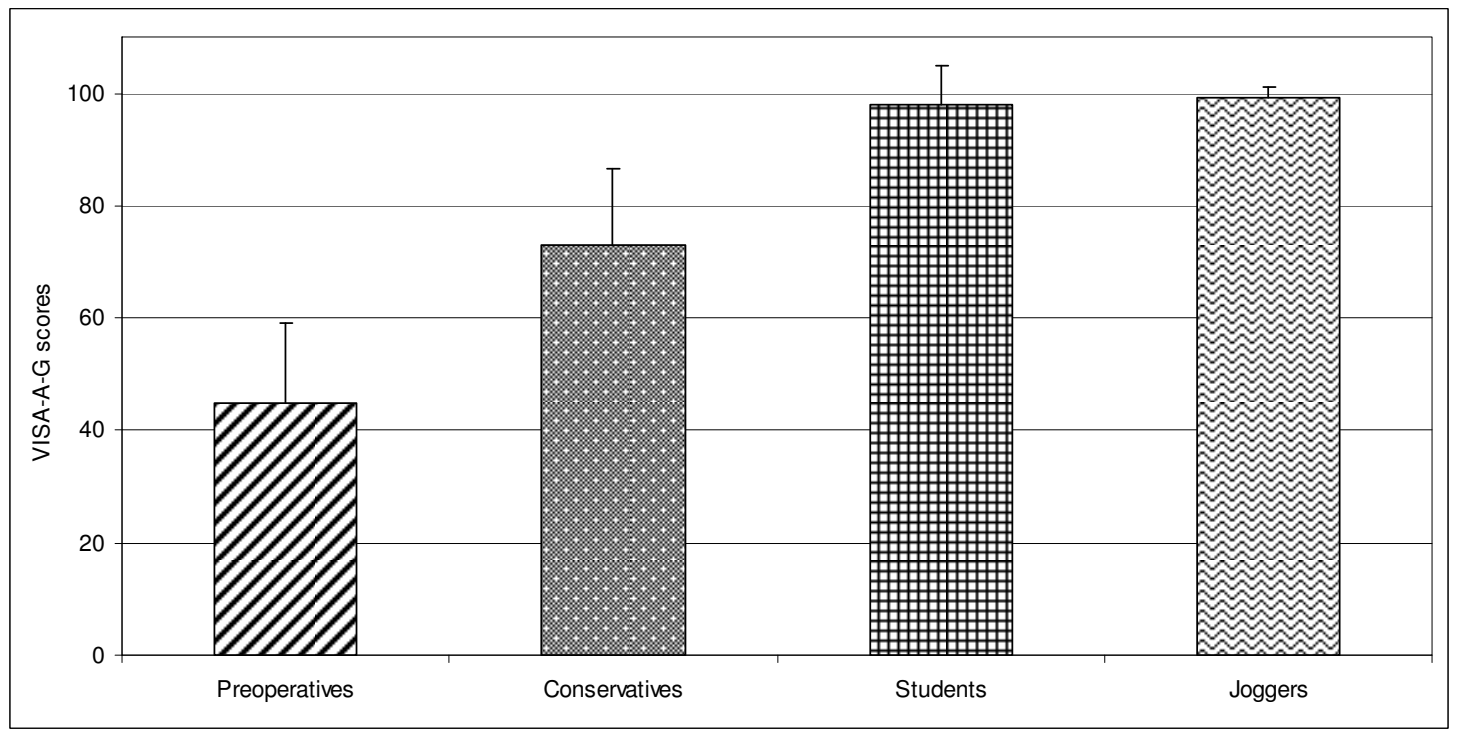

Figure I

Bar chart demonstrating the VISA-A-G mean scores (plus standard deviation) in relation to the tested groups.

the Percy and Conochie tendon classification [19] Spearman's rho was 0.95 ( $\mathrm{p}=0.000$; Figure 2). Spearman's rho was determined to be $\quad-0.95(\mathrm{p}=0.000)$ when VISAA-G questionnaire and the "classification system for the effect of pain on athletic performance" [20] were compared (Figure 3).

\section{Reliability testing}

Reliability testing revealed no relevant difference when subsets of participants of the study were evaluated by the VISA-A-G questionnaire two times within one week (ICC
$=0.60$ to 0.97 ; Table 3$)$. This was also true for reliability analysis of the individual test items 1, 2, 3, 4, 5, 7 and 8 (ICC $=0.65$ to 0.89 ; Table 4 ). For question 6 ICC was 0.44 $(\mathrm{p}=0.159)$. Interobserver agreement was excellent $(\mathrm{n}=$ 10; Pearson $\mathrm{r}=0.97, \mathrm{p}=0.000 ; \mathrm{ICC}=0.99, \mathrm{p}=0.000)$.

\section{Discussion}

Results of self-administered questionnaires in "populations divided by language or by culture" may systematically be biased if not equivalent in the original and target

Table 2: Results of VISA-A-G ratings from the present study compared with the original English publication [8].

\begin{tabular}{|c|c|c|c|c|c|c|}
\hline & & & \multicolumn{4}{|c|}{ Present study } \\
\hline & & & \multicolumn{2}{|c|}{ Healthy } & \multicolumn{2}{|c|}{ Patients } \\
\hline & & & $\begin{array}{l}\text { Students } \\
98 \pm 7\end{array}$ & $\begin{array}{l}\text { Joggers } \\
99 \pm 2\end{array}$ & $\begin{array}{c}\text { Conservatives } \\
73 \pm 142\end{array}$ & $\begin{array}{c}\text { Preoperatives } \\
45 \pm 14\end{array}$ \\
\hline & & $\mathrm{n}$ & 48 & 31 & 15 & 15 \\
\hline \multirow[t]{4}{*}{$\begin{array}{c}\text { Robinson } \\
\text { et al. }[]\end{array}$} & $\begin{array}{l}\text { Healthy } \\
96 \pm 7\end{array}$ & 63 & 0.138 & & & \\
\hline & $\begin{array}{l}\text { Joggers } \\
98 \pm 3\end{array}$ & 20 & & 0.198 & & \\
\hline & $\begin{array}{c}\text { Conservatives } \\
64 \pm 17\end{array}$ & 45 & & & 0.050 & \\
\hline & $\begin{array}{c}\text { Preoperatives } \\
44 \pm 28\end{array}$ & 14 & & & & 0.905 \\
\hline
\end{tabular}




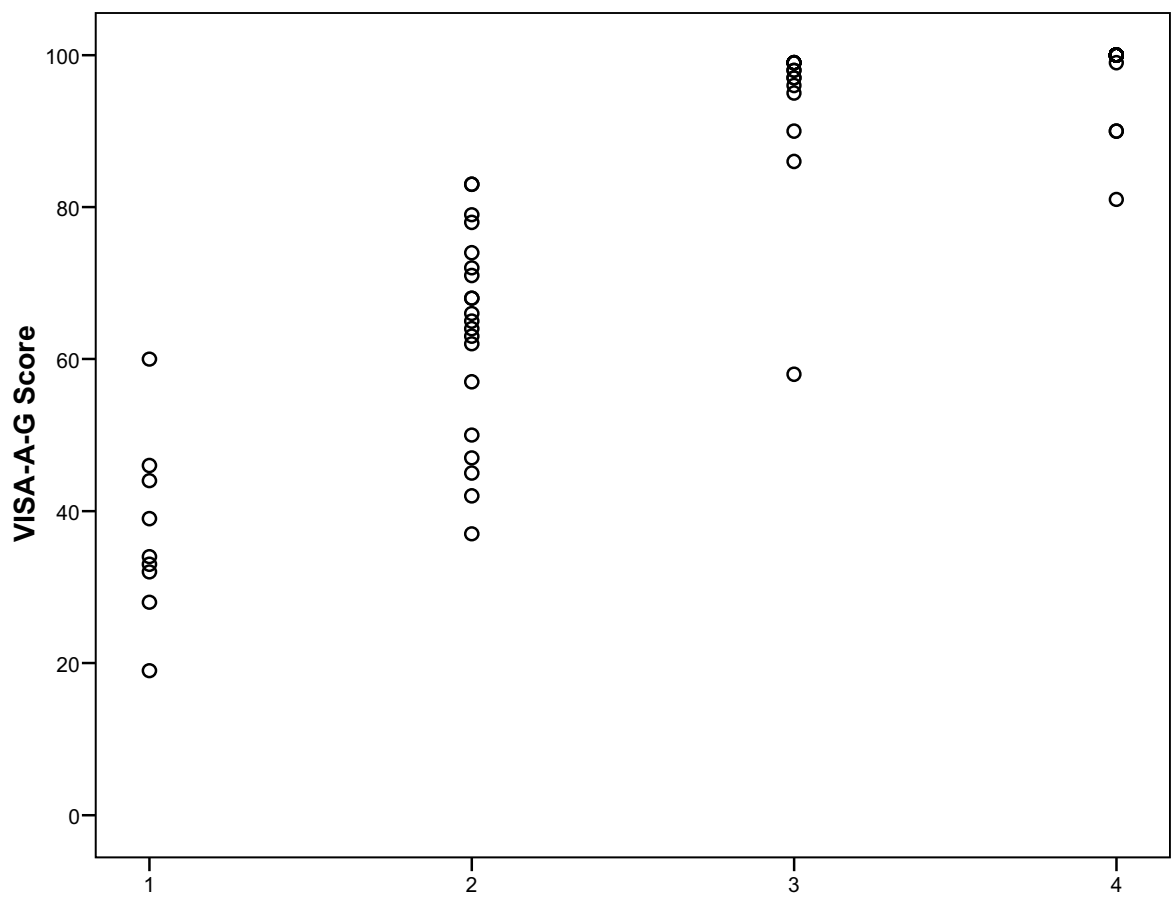

Figure 2

Relation between VISA-A-G score and Achilles tendon grade of severity by Percy and Conochie [19]. I = excellent (full function and no residual disability); 2 = good (no real limitation of activities); 3 = fair (some limitation of activities); 4 = poor (severe weakness and marked limp).

version [17]. Therefore it was necessary to adapt the VISAA score also to a German version.

The most important finding of the present study is that the VISA-A score was successfully adapted to a German version and this is introduced. Reliability and validity have been tested showing moderate to excellent results.

Systematic stepwise translation and cross-cultural adaptation of the VISA-A questionnaire into a German version (VISA-A-G) was successfully performed. To be most accurate and comparable this process was performed by following the guidelines developed by Beaton et al. as close as possible [17]. Summarizing the results of the six stages of cross-cultural adaptation only question 8 caused some confusion to the forward and back translators and during the expert committee meeting as well. If someone has no sport related Achilles tendon pain and scores 0 min of sports ability, the reason not to train cannot be related to Achilles tendon pain but may be due to low general fitness. We discussed this point with the authors of the original VISA-A questionnaire by E-mail. Besides considering that different answering modalities have been introduced to enable pain free patients to score their actual level of Achilles tendon rehabilitation we were notified that "in practice" they "haven't had any problems with this" (Karim Khan personal communication, 2009).

During VISA-A-G questionnaire pretesting we realized that some subjects got into trouble marking the correct question 1 answering boxes. Analogue to the VISA-A-S cross-cultural adaptation [9] we facilitated filling out this question by inserting the minutes in each respective box in the final VISA-A-G version.

For the validation and reliability testing we implemented the methods which were described in the original publication [8]. Showing significant $(p=-0.95$ and 0.95$)$ correlations between the VISA-A-G questionnaire and the two compared classification systems in all tested groups concurrent validity testing of the VISA-A-G questionnaire is considered to be excellent. Presenting ICC values from 0.60 to 0.97 also the reliability testing was proven to be "moderate" to "accurate" [21] with respect to each individual group of test subjects. It is unclear, why question 6 could not be proven to be sufficiently reliable. VISA-A-G questionnaire results are not biased by different observers. This is a result of the self administered and easy to use nature of the questionnaire. Face validity as assessed by the observers was good. From our experience with the test- 


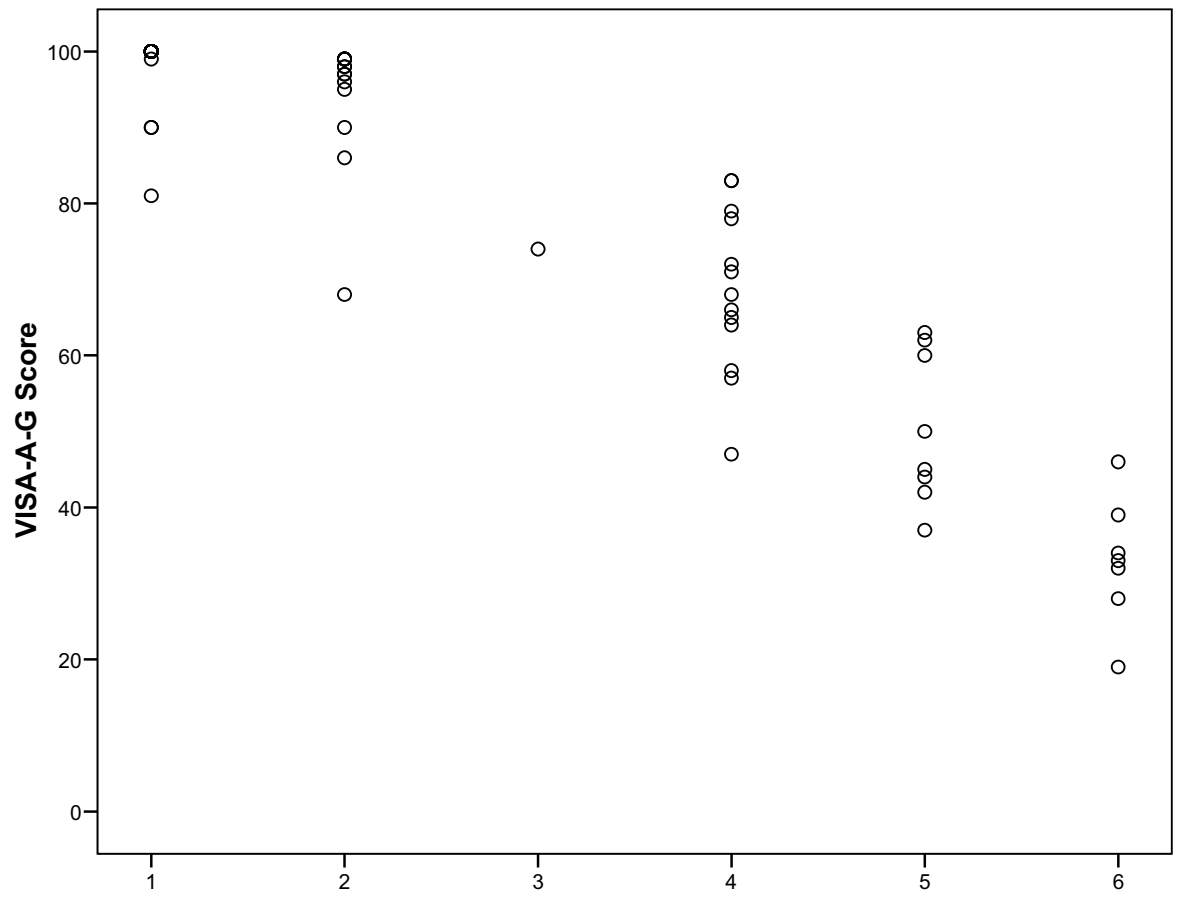

Figure 3

Relation between VISA-A-G score and the "classification system for the effect of pain on athletic performance" by Curwin and Stanish [20]. I = no pain and unrestricted level of sports performance; 2 = pain only with extreme exertion and unrestricted level of sports performance; 3 = pain with extreme exertion and I to 2 hours afterward and normal or slightly decreased level of sports performance; 4 = pain during and after any vigorous activities and somewhat decreased level of sports performance; 5 = pain during activity forcing termination and markedly decreased level of sports performance; 6 $=$ pain during daily activities and unable to perform sport.

ing procedure we feel that it can enable also postal explorations.

The authors of the original VISA-A questionnaire emphasize that it "is not a diagnostic tool" nor does it play a role in decision making for Achilles tendon surgery [8] and this statement is true also for the respective translations including the VISA-A-G questionnaire. Specific local pathologies (different foot and ankle disorders), central or peripheral sciatic nerve lesions have to been ruled out as differential diagnoses before administering one of the VISA-A questionnaires.

Included pathologies are different from previous work on this topic. Contrasting to the original VISA-A publication and to its Swedish translation $[8,9]$ but according to the Italian translation [12] patients suffering from Haglund's disease and insertional tendinopathy were excluded. We believe that these are completely separate pathoanatomic entities [22] and its inclusion possibly may bias the results. Therefore the data presented for the validation and reliability testing of the VISA-A-G questionnaire are valid only for Achilles tendinopathy. A separate validation procedure is recommended for Haglund's disease and insertional Achilles tendinopathy patients. Also different from the original VISA-A publication [8] and from the Swedish translation [9] we excluded patients with bilateral Achilles tendon symptoms from participating in the study as this could possibly confound the answering behaviour.

The VISA-A questionnaire is a universally accepted instrument. In a Medline search 23 publications were found entering "VISA-A" in the "all fields" category. Of these eight were randomized and controlled trials and used the VISA-A questionnaire $(\mathrm{n}=6)$ or the VISA-A-S (Swedish version) questionnaire $(n=2)$. There were two cross-cultural VISA-A adaptations [9,12]. Analyzing the methodological details, however, five of the randomized controlled investigations were performed (at least in part) in not English speaking countries and VISA-A questionnaires which are not cross-culturally adapted were used [10,11,13-15]. Even when published in high ranked and reviewed journals the results obtained from these studies 
Table 3: Results of reliability testing of the VISA-A-G questionnaire.

\begin{tabular}{|c|c|c|c|c|c|c|}
\hline & Spearman's rho & $\mathbf{p}$ & ICC & $\mathbf{p}$ & Wilcoxon & Cronbach's alpha \\
\hline Test-Retest Conservative Group $(n=15)$ & 0.66 & 0.007 & 0.87 & 0.000 & 0.442 & \\
\hline Test-Retest Students Group $(n=48)$ & 0.60 & 0.000 & 0.97 & 0.000 & 0.011 & \\
\hline Test-Retest Joggers Group $(n=31)$ & 0.70 & 0.000 & 0.60 & 0.033 & 0.020 & \\
\hline Internal consistency Patients Group $(n=30)$ & & & & & & 0.74 \\
\hline
\end{tabular}

$\mathrm{ICC}=$ Intraclass Correlation Coefficient.

are likely biased "as translation does not automatically provide a valid measure of another culture's health" [17]. The VISA-A score is developing to be the gold standard to determine the clinical severity of painful Achilles tendon lesions. Further cross-cultural adapted and validated versions of the VISA-A questionnaire are needed especially when international study groups from different language speaking countries cooperate and compare their results.

The present study explores data on concurrent validity testing of the VISA-A-G questionnaire and compares the results in groups of university students, uninjured joggers, conservatively treated, and preoperative patients with the published results from the original publication (VISA-A). Responsiveness including the minimal detectable change and the minimal clinically important difference have to be evaluated in future investigations.

\section{Conclusion}

The VISA-A questionnaire was successfully cross-cultural adapted and validated for use in German speaking popu-

Table 4: Results of reliability testing of the eight VISA-A-G items.

\begin{tabular}{lccccc}
\hline & Spearman's rho & p & ICC & p & Wilcoxon \\
\hline Question 1 & 0.58 & 0.022 & 0.89 & 0.000 & 0.916 \\
\hline Question 2 & 0.89 & 0.000 & 0.89 & 0.000 & 0.942 \\
\hline Question 3 & 0.64 & 0.009 & 0.53 & 0.071 & 0.088 \\
\hline Question 4 & 0.65 & 0.009 & 0.69 & 0.022 & 0.565 \\
\hline Question 5 & 0.56 & 0.031 & 0.65 & 0.023 & 0.223 \\
\hline Question 6 & 0.23 & 0.411 & 0.44 & 0.159 & 0.681 \\
\hline Question 7 & 0.69 & 0.004 & 0.84 & 0.001 & 0.141 \\
\hline Question 8 & 0.65 & 0.009 & 0.75 & 0.007 & 0.279 \\
\hline
\end{tabular}

ICC = Intraclass Correlation Coefficient. lations. The psychometric properties of the VISA-A-G questionnaire are similar to those of the original English and the Swedish version. It therefore can be recommended as a sufficiently robust tool for future measuring clinical severity of Achilles tendinopathy in German speaking patients.

\section{Competing interests}

The authors declare that they have no competing interests.

\section{Authors' contributions}

HL conceived the study, participated in its design, performed data acquisition, interpreted the data and drafted the manuscript. TN conceived of the study, participated in its design, performed data acquisition, analyzed and interpreted the data and helped to draft the manuscript. Both authors read and approved the final manuscript.

\section{Additional material}

\section{Additional file 1}

Final version of the VISA-A-G questionnaire. translated and cross-culturally adapted VISA-A-G questionnaire.

Click here for file

[http://www.biomedcentral.com/content/supplementary/14712474-10-134-S1.doc]

\section{Acknowledgements}

The authors gratefully acknowledge the contribution of Dr. Ackermann, Department of Medical Statistics, University Frankfurt for his expert advice and Prof. Karim Khan, PhD Karin Grävare Silbernagel, and PhD Dorcas E. Beaton for their valuable conceptual E-mail discussions during the crosscultural adaptation and validation process of the VISA-A-G questionnaire. We further thank Michael Bartelt (forward translation), Michael Shields (back translation, Patty Schütze (back translation, expert committee), Christiane Riedel (expert committee), and Robin Limmeroth (expert committee) for their relevant contributions. The article processing charge has kindly be accepted by Orthomol pharmazeutische Vertriebs GmbH, Langenfeld, Germany 


\section{References}

I. Lohrer $\mathrm{H}$ : [Rare causes and differential diagnoses of Achilles tendinitis]. Sportverletz Sportschaden 1991, 5: I82-185.

2. Schepsis AA, Jones $H$, Haas AL: Achilles tendon disorders in athletes. Am J Sports Med 2002, 30:287-305.

3. Maffulli N, Khan KM, Puddu G: Overuse tendon conditions: time to change a confusing terminology. Arthroscopy 1998, | 4:840-843.

4. Paavola M, Kannus P, Paakkala T, Pasanen M, Jarvinen M: Long-term prognosis of patients with achilles tendinopathy. An observational 8-year follow-up study. Am J Sports Med 2000, 28:634-642.

5. Myerson MS, McGarvey W: Disorders of the insertion of the Achilles tendon and Achilles tendinitis. J Bone Joint Surg Am |998, 80-A: |8|4-|824.

6. Knobloch K, Yoon U, Vogt PM: Acute and overuse injuries correlated to hours of training in master running athletes. Foot Ankle Int 2008, 29:67I-676.

7. Andres BM, Murrell GA: Treatment of tendinopathy: what works, what does not, and what is on the horizon. Clin Orthop Relat Res 2008, 466:1539-1554.

8. Robinson JM, Cook JL, Purdam C, Visentini PJ, Ross J, Maffulli N, Taunton JE, Khan KM: The VISA-A questionnaire: a valid and reliable index of the clinical severity of Achilles tendinopathy. Br J Sports Med 200I, 35:335-34I.

9. Silbernagel KG, Thomee R, Karlsson J: Cross-cultural adaptation of the VISA-A questionnaire, an index of clinical severity for patients with Achilles tendinopathy, with reliability, validity and structure evaluations. BMC Musculoskelet Disord 2005, 6:12.

10. de Jonge S, de Vos RJ, van Schie HT, Verhaar JA, Weir A, Tol JL: Oneyear follow-up of a randomised controlled trial on added splinting to eccentric exercises in chronic midportion Achilles tendinopathy. Br J Sports Med 2008 in press.

II. de Vos RJ, Weir A, Visser RJ, de WT, Tol JL: The additional value of a night splint to eccentric exercises in chronic midportion Achilles tendinopathy: a randomised controlled trial. $\mathrm{Br} J$ Sports Med 2007, 4I:e5.

12. Maffulli N, Longo UG, Testa V, Oliva F, Capasso G, Denaro V: Italian translation of the VISA-A score for tendinopathy of the main body of the Achilles tendon. Disabil Rehabil 2008, 30:1635-1639.

13. Rompe JD, Nafe B, Furia JP, Maffulli N: Eccentric loading, shockwave treatment, or a wait-and-see policy for tendinopathy of the main body of tendo Achillis: a randomized controlled trial. Am J Sports Med 2007, 35:374-383.

I4. Rompe JD, Furia J, Maffulli N: Eccentric Loading Versus Eccentric Loading Plus Shock-Wave Treatment for Midportion Achilles Tendinopathy: A Randomized Controlled Trial. Am J Sports Med 2008, 37(3):463-70.

15. Rompe JD, Furia J, Maffulli N: Eccentric loading compared with shock wave treatment for chronic insertional achilles tendinopathy. A randomized, controlled trial. J Bone Joint Surg Am 2008, 90:52-61.

16. Silbernagel KG, Thomee R, Eriksson BI, Karlsson J: Continued sports activity, using a pain-monitoring model, during rehabilitation in patients with Achilles tendinopathy: a randomized controlled study. Am J Sports Med 2007, 35:897-906.

17. Beaton DE, Bombardier C, Guillemin F, Ferraz MB: Guidelines for the process of cross-cultural adaptation of self-report measures. Spine 2000, 25:3186-3191.

18. Halasi T, Kynsburg A, Tallay A, Berkes I: Development of a new activity score for the evaluation of ankle instability. $\mathrm{Am} J$ Sports Med 2004, 32:899-908.

19. Percy EC, Conochie LB: The surgical treatment of ruptured tendo achillis. Am J Sports Med 1978, 6:132-136.

20. Curwin S, Stanish WD: Tendinitis: Its etiology and treatment Lexington: Collamore Press; 1984.

21. Kerkhoffs GM, Blankevoort L, van Dijk CN: A measurement device for anterior laxity of the ankle joint complex. Clin Biomech (Bristol, Avon) 2005, 20:2 18-222.

22. Lohrer H, Arentz S, Nauck T, Dorn-Lange NV, Konerding MA: The Achilles tendon insertion is crescent-shaped: an in vitro anatomic investigation. Clin Orthop Relat Res 2008, 466:2230-2237.

\section{Pre-publication history}

The pre-publication history for this paper can be accessed here: http://www.biomedcentral.com/1471-2474/10/134/pre pub
Publish with Biomed Central and every scientist can read your work free of charge

"BioMed Central will be the most significant development for disseminating the results of biomedical research in our lifetime. "

Sir Paul Nurse, Cancer Research UK

Your research papers will be:

- available free of charge to the entire biomedical community

- peer reviewed and published immediately upon acceptance

- cited in PubMed and archived on PubMed Central

- yours - you keep the copyright

Submit your manuscript here:

http://www.biomedcentral.com/info/publishing_adv.asp
BioMedcentral 